\section{Estudo dos determinantes clínicos e epidemiológicos das concentrações de sódio e potássio no colostro de nutrizes hipertensas e normotensas}

\author{
Clinical and epidemiological determinants of \\ sodium and potassium levels in the colostrum \\ of nursing mothers with and without \\ hypertension in Brazil
}

Marília Alfenas de Oliveira Sírio 1 Marcelo Eustáquio Silva 2

Heberth de Paula 2

Maria Cristina Passos 2

Antônio Otávio de Souza Sobrinho 2
1 Sociedade Beneficente São Camilo, Hospital Monsenhor Horta, Mariana, Brasil. 2 Escola de Nutrição, Universidade Federal de Ouro Preto, Ouro Preto, Brasil.

Correspondência M. E. Silva

Departamento de Alimentos Escola de Nutrição, Universidade de Ouro Preto. Morro do Cruzeiro, Campus Universitário, Ouro Preto, $M G$ 35400-000, Brasil. mesilva@enut.ufop.br

\begin{abstract}
This study aimed to determine whether maternal hypertension and other clinical and epidemiological determinants have an impact on sodium and potassium levels in the 48-hour colostrum of breastfeeding mothers. The study included 105 randomly selected breastfeeding mothers, of whom 72 (68.8\%) had normal blood pressure and 33 (31.4\%) were hypertensive. Colostrum was collected in-hospital in the morning, and sodium and potassium concentrations were measured using a flame photometer. When sodium and potassium concentrations were compared to the variables age, parity, family history of hypertension, gestational age, birth weight, and socioeconomic factors, colostrum potassium level was associated with maternal age. Average sodium and potassium levels in 48hour colostrum non-hypertensive and hypertensive breastfeeding mothers showed no significant differences. Thus, breastfeeding by hypertensive mothers probably does not increase the risk of their children developing hypertension in adulthood.
\end{abstract}

Colostrum; Hypertension; Sodium; Potassium

\section{Introdução}

A hipertensão arterial, uma doença crônico-degenerativa de grande prevalência na população mundial, vem, na atualidade, alcançando elevadas taxas de morbi-mortalidade. Sua etiologia passa por fatores genéticos e ambientais, dentre estes a nutrição humana. Já é fato cientificamente comprovado que dietas ricas em sódio, aliadas ao baixo consumo de potássio, desencadeiam a hipertensão arterial em indivíduos geneticamente predispostos.

Em se tratando de nutrição, o primeiro alimento natural destinado à espécie humana logo após o nascimento é o leite materno, particularmente denominado colostro - um líquido amarelado, espesso, secretado em pequenos volumes ( 2 a 20mL por mamada) nos primeiros dias pósparto, mas suficiente para garantir a nutrição, hidratação e proteção do recém-nascido.

Inúmeros trabalhos já investigaram a composição química do leite materno, entretanto poucos trabalhos têm investigado a possível relação do leite materno, e mais especificamente do colostro, a doenças crônico-degenerativas. Vale ressaltar trabalhos como o de Hoffman et al. 1, que verificaram que crianças alimentadas com dieta pobre em sódio desde o nascimento, tiveram pressões arteriais significativamente menores aos seis meses de vida que aquelas alimentadas com fórmulas artificiais desde o nascimento. 
Holliday et al. 2 realizaram um estudo com ratas nutrizes hipertensas e normotensas e observaram que filhotes de ratas predispostas à hipertensão, quando amamentados por ratas nutrizes normotensas, tornaram-se menos hipertensos na idade adulta que aqueles amamentados por ratas nutrizes hipertensas. Isso devido, em parte, ao maior teor de sódio e mais alta razão $\mathrm{Na} / \mathrm{K}$ que caracterizam o leite das ratas nutrizes hipertensas.

Estudos epidemiológicos de hipertensão arterial na infância têm sido fonte importante de subsídios, fornecendo indícios de que a hipertensão arterial sistêmica do adulto começa na infância 3 . Nesse contexto, poderia o colostro de mulheres hipertensas conter níveis de sódio e potássio inapropriados à nutrição do recémnascido, expondo-o à condição de risco para a hipertensão arterial na vida futura?

Para avaliar possíveis associações da hipertensão materna e de outras variáveis clínicas e epidemiológicas com as concentrações de sódio e potássio no colostro de 48 horas de mulheres nutrizes, realizou-se o presente estudo.

\section{Material e métodos}

Este é um estudo de delineamento transversal e natureza quantitativa, envolvendo 105 puérperas nutrizes de um hospital do Estado de Minas Gerais, Brasil. Foram convidadas a participar do estudo todas as nutrizes hipertensas e, dentre as normotensas, aquelas cujos números de registro de entrada no bloco obstétrico foram múltiplos de 5. Na composição dos grupos de nutrizes foram incluídas apenas aquelas que deram seu consentimento, livre, esclarecido e por escrito, manifestando sua participação espontânea e voluntária, Foram excluídas as nutrizes que eram tabagistas, que apresentaram gestação múltipla e intercorrências no pré e pós-parto. Este estudo foi aprovado pelo Comitê de Ética em Pesquisa da Universidade Federal de Ouro Preto.

As nutrizes foram selecionadas para o grupo das normotensas ( $\mathrm{n}=72 ; 68,6 \%$ ) ou para o das hipertensas ( $n=33 ; 31,4 \%)$, dentre estas: as portadoras de hipertensão arterial sistêmica crônica (HASc, $\mathrm{n}=1 ; 3 \%$ ), as que apresentaram doença hipertensiva específica da gestação (DHEG, $\mathrm{n}=$ $27 ; 81,8 \%$ ) ou que apresentaram a DHEG superposta à HASc ( $n=5 ; 15,2 \%)$, conforme valores de pressões arteriais aferidas à internação e diagnóstico clínico relatado em prontuário médico.

As pressões arteriais foram aferidas à internação e no dia da coleta do colostro por pessoal da enfermagem devidamente treinado. A coleta do colostro ( 2 a $5 \mathrm{~mL}$ ) foi realizada nas 48 horas pós- parto pela nutriz sob a supervisão da autora (M. A. O. S.), ou pela própria autora. As amostras de colostro foram congeladas e posteriormente encaminhadas ao Laboratório de Química, Universidade Federal de Ouro Preto para preparo: centrifugação e diluição a 1:10. Para determinação das concentrações de sódio e potássio utilizou-se o fotômetro de chama (marca Micronal B-462, São Paulo, Brasil) no Laboratório de Química da Universidade Federal de Ouro Preto. Para checagem dos dados, amostras aleatórias de colostro foram enviadas ao hospital para determinação das concentrações de sódio e potássio. A dieta das nutrizes durante o período de internação foi normossódica e normocalêmica (aproximadamente $2.400 \mathrm{mg}$ de sódio e $3.000 \mathrm{mg}$ de potássio ao dia), prescrita pela autora (M. A. O. S.).

Utilizou-se um formulário de entrevista estruturada para obtenção dos seguintes dados: idade, paridade, história familiar de hipertensão arterial sistêmica e indicadores sócio-econômicos (renda familiar bruta em salários mínimos, procedência, freqüência ao pré-natal e acesso a orientações para o sucesso do aleitamento materno). Dados como idade gestacional na ocasião do parto, peso do recém-nascido, pressão arterial de internação e pressão arterial no dia da coleta do colostro foram obtidos dos prontuários médicos das nutrizes e dos seus respectivos neonatos.

Os dados foram armazenados em um banco criado no programa Epi Info 6.04d (Centers for Disease Control and Prevention, Atlanta, Estados Unidos), e testes para comparação das médias de pressão arterial, idade, peso ao nascer e das concentrações de sódio e potássio obtidas dos dois grupos de nutrizes (teste $t$ de student e análise de variância - ANOVA) e de testes de associações entre as variáveis independentes e as concentrações de sódio e potássio (qui-quadrado, correlação de Pearson e análise multivariada) foram realizados. Adotou-se como faixa de normalidade para as concentrações de sódio no colostro valores de 5 a $85 \mathrm{mmol} / \mathrm{L}$, conforme achados de Grumash et al. ${ }^{4}$, e para as concentrações de potássio no colostro valores de 12 a $25 \mathrm{mmol} / \mathrm{L}$, segundo achados de Gross et al. 5 . Esses estudos foram selecionados em função do período lactacional em que se coletou o leite materno ter sido semelhante ao do presente estudo, ou seja, leite materno nas primeiras 48 horas - fase de colostro. Considerou-se significância estatística quando $\mathrm{p}$ assumiu valores $\leq$ 0,05 . 


\section{Resultados}

A idade média das nutrizes adultas normotensas foi $26,4 \pm 5,5$ anos e das adultas hipertensas $29,7 \pm$ 6,6 . A idade média das nutrizes adolescentes normotensas foi $17,2 \pm 1,3$ anos e a idade da única nutriz adolescente hipertensa foi 17 anos.

Na Tabela 1 verifica-se que, dentre as $105 \mathrm{nu}-$ trizes participantes do estudo, 27 (25,7\%) eram adolescentes (idades de 10 a 19 anos segundo a OMS 6 ) e 78 (74,3\%) eram adultas (idade igual ou superior a 20 anos). Quanto à paridade, 6 $(18,2 \%)$ eram nutrizes primíparas hipertensas e 27 (81,8\%) eram multíparas hipertensas. Quanto à história familiar de hipertensão, 20 (60,6\%) das nutrizes hipertensas e 33 (45,8\%) das normotensas relataram história materna de hipertensão e $10(30,3 \%)$ das nutrizes hipertensas e 22 (30,5\%) das normotensas relataram história paterna de hipertensão. Dentre as hipertensas, 7 (21,2\%) tinham ambos os pais hipertensos e dentre as normotensas, $11(15,3 \%)$ tinham ambos os pais hipertensos. Analisando-se a idade gestacional, $18(17,1 \%)$ nutrizes do estudo tiveram gestação pré-termo, sendo que $13(18,1 \%)$ cujos partos foram pré-termos eram normotensas e $5(15,2 \%)$ eram hipertensas. Com relação ao peso ao nascer do recém-nascido, $81(77,1 \%)$ nutrizes tiveram

Tabela 1

Caracterização das nutrizes normotensas e hipertensas do estudo.

\begin{tabular}{|c|c|c|c|c|c|c|}
\hline \multirow[t]{2}{*}{ Variáveis } & \multicolumn{2}{|c|}{ Freqüência total } & \multicolumn{2}{|c|}{ Normotensas } & \multicolumn{2}{|c|}{ Hipertensas } \\
\hline & $\mathbf{N}$ & $\%$ & $\mathbf{n}$ & $\%$ & $n$ & $\%$ \\
\hline \multicolumn{7}{|l|}{ Idade } \\
\hline Adolescente & 27 & 25,7 & 26 & 36,1 & 1 & 3,0 \\
\hline Adulta & 78 & 74,3 & 46 & 63,9 & 32 & 97,0 \\
\hline \multicolumn{7}{|l|}{ Paridade } \\
\hline Primípara & 45 & 42,9 & 39 & 54,2 & 6 & 18,2 \\
\hline Multípara & 60 & 57,1 & 33 & 45,8 & 27 & 81,8 \\
\hline \multicolumn{7}{|c|}{ História familiar de HAS * } \\
\hline Mãe com HAS & 53 & 50,5 & 33 & 45,8 & 20 & 60,6 \\
\hline Mãe sem HAS & 48 & 45,7 & 38 & 52,8 & 10 & 30,3 \\
\hline Pai com HAS & 32 & 30,5 & 22 & 30,6 & 10 & 30,3 \\
\hline Pai sem HAS & 69 & 65,7 & 49 & 68,1 & 20 & 60,6 \\
\hline Ambos com HAS & 18 & 17,1 & 11 & 15,3 & 7 & 21,2 \\
\hline \multicolumn{7}{|c|}{ Idade gestacional no parto } \\
\hline Pré-termo & 18 & 17,1 & 13 & 18,1 & 5 & 15,2 \\
\hline A termo & 87 & 82,9 & 59 & 81,9 & 28 & 84,8 \\
\hline \multicolumn{7}{|l|}{ Peso ao nascer } \\
\hline Baixo peso & 24 & 22,9 & 17 & 23,6 & 7 & 21,2 \\
\hline Peso normal & 81 & 77,1 & 55 & 76,4 & 26 & 78,8 \\
\hline \multicolumn{7}{|l|}{ Freqüência ao pré-natal } \\
\hline Sim & 101 & 96,2 & 68 & 94,4 & 33 & 100 \\
\hline Não & 4 & 3,8 & 4 & 5,6 & 0 & \\
\hline \multicolumn{7}{|c|}{ Renda familiar (salário mínimo) * } \\
\hline$<1$ & 37 & 35,2 & 27 & 37,5 & 10 & 30,3 \\
\hline $1-2$ & 31 & 29,5 & 19 & 26,4 & 12 & 36,4 \\
\hline$>2$ & 33 & 31,4 & 23 & 31,9 & 10 & 30,3 \\
\hline \multicolumn{7}{|c|}{ Acesso a orientação para o sucesso do aleitamento materno } \\
\hline Sim & 99 & 94,3 & 68 & 94,4 & 31 & 93,9 \\
\hline Não & 6 & 5,7 & 4 & 5,6 & 2 & 6,1 \\
\hline \multicolumn{7}{|l|}{ Procedência } \\
\hline Urbana & 72 & 68,6 & 50 & 69,4 & 22 & 66,7 \\
\hline Rural & 33 & 31,4 & 22 & 30,6 & 11 & 33,3 \\
\hline
\end{tabular}

HAS: hipertensão arterial sistêmica.

* Quatro nutrizes não souberam informar acerca de história familiar de hipertensão e renda familiar. 
seus bebês com peso normal (igual ou superior a 2.500g). Dentre as nutrizes hipertensas ( $\mathrm{n}=$ 33), 7 (21,2\%) tiveram seus bebês com baixo peso ao nascer e dentre as normotensas $(n=72)$, 17 (23,6\%) tiveram seus bebês com baixo peso ao nascer. Quando se compararam as médias de peso ao nascer dos recém-nascidos de nutrizes normotensas e hipertensas, não se encontrou diferença significativa. Quanto aos aspectos do pré-natal, 101 (96,2\%) compareceram ao exame e, dentre estas, encontram-se $100 \%$ das nutrizes hipertensas; 99 (94,3\%) receberam orientações para o sucesso no aleitamento materno, dentre estas 31 (96,9\%) nutrizes hipertensas. Quanto à renda familiar em termos de salários mínimos, observa-se porcentuais bastante próximos, sendo que 37 (35,2\%) das nutrizes recebem menos de um salário mínimo mensal. Quanto à procedência das nutrizes, o porcentual de hipertensas provenientes da zona urbana foi o dobro daquelas hipertensas provenientes da zona rural $(66,7 \%$ e 33,3\%, respectivamente).

$\mathrm{Na}$ Tabela 2, a associação entre as variáveis maternas: idade, paridade, história familiar de hipertensão, peso ao nascer do recém-nascido, idade gestacional na ocasião do parto, freqüên-

Associação entre as variáveis maternas peso ao nascer, aspectos do pré-natal e situação sócio-econômica, e a presença ou ausência de hipertensão arterial nas nutrizes do estudo.

\begin{tabular}{|c|c|c|c|c|c|c|c|}
\hline & \multicolumn{4}{|c|}{ Hipertensão } & \multirow[t]{2}{*}{$\chi^{2}$} & \multirow[t]{2}{*}{ 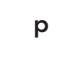 } & \multirow[t]{2}{*}{ OR } \\
\hline & Sim & $\%$ & Não & $\%$ & & & \\
\hline \multicolumn{8}{|l|}{ Idade } \\
\hline Adulta & 32 & 97,0 & 46 & 63,9 & 11,29 & 0,001 & 18,09 \\
\hline Adolescente & 1 & 3,0 & 26 & 36,1 & & & $(2,36-382,38)$ \\
\hline \multicolumn{8}{|l|}{ Paridade } \\
\hline Primípara & 6 & 18,2 & 39 & 54,2 & 10,54 & 0,001 & 0,19 \\
\hline Multípara & 27 & 81,8 & 33 & 45,8 & & & $(0,06-0,56)$ \\
\hline \multicolumn{8}{|c|}{ História familiar de HAS } \\
\hline Mãe hipertensa & 20 & 60,6 & 33 & 45,8 & & & \\
\hline Mãe normotensa & 10 & 30,3 & 38 & 52,8 & & & \\
\hline Pai hipertenso & 10 & 30,3 & 22 & 30,6 & & & \\
\hline Pai normotenso & 20 & 60,6 & 49 & 68,1 & & & \\
\hline \multicolumn{8}{|c|}{ Peso do recém-nascido } \\
\hline Peso normal & 26 & 78,8 & 55 & 76,4 & & & \\
\hline Baixo peso & 7 & 21,2 & 17 & 23,6 & & & \\
\hline \multicolumn{8}{|l|}{ Idade gestacional } \\
\hline Pré-termo & 5 & 15,2 & 13 & 18,1 & & & \\
\hline A termo & 28 & 84,8 & 59 & 81,9 & & & \\
\hline \multicolumn{8}{|l|}{ Pré-natal } \\
\hline $\operatorname{Sim}$ & 33 & 100,0 & 68 & 94,4 & & & \\
\hline Não & 0 & 0,0 & 4 & 5,6 & & & \\
\hline \multicolumn{8}{|c|}{ Orientações sobre aleitamento materno } \\
\hline $\operatorname{Sim}$ & 31 & 93,9 & 68 & 94,4 & & & \\
\hline Não & 2 & 6,1 & 4 & 66,7 & & & \\
\hline \multicolumn{8}{|c|}{ Renda (salário mínimo) * } \\
\hline$<1$ & 10 & 30,3 & 27 & 37,5 & & & \\
\hline $1-2$ & 12 & 36,4 & 19 & 26,4 & & & \\
\hline$>2$ & 10 & 30,3 & 23 & 31,9 & & & \\
\hline \multicolumn{8}{|l|}{ Procedência } \\
\hline Urbana & 22 & 66,7 & 50 & 69,4 & & & \\
\hline Rural & 11 & 33,3 & 22 & 30,6 & & & \\
\hline
\end{tabular}

p: nível de significância estatística do teste do $\chi^{2}$, sendo $\mathrm{p}<0,05$ indicativo de significância estatística; OR: odds ratio; IC: intervalo de confiança é estatisticamente significativo no nível de $\mathrm{p}<0,05$ quando 1 não estiver dentro do intervalo; HAS: hipertensão arterial sistêmica.

* $3,8 \%$ (4) das nutrizes não souberam informar acerca da história familiar de hipertensão e renda. 
cia ao pré-natal, acesso às orientações para o sucesso do aleitamento materno, renda familiar e procedência das nutrizes, foram testadas e apenas a idade e paridade mostraram associação significativa com a presença de hipertensão nas nutrizes.

Na Tabela 3 é mostrada a freqüência com que as nutrizes, caracterizadas pelas diferentes variá- veis: ser ou não portadora de hipertensão, idade, paridade, história familiar materna e paterna de hipertensão arterial sistêmica, idade gestacional na ocasião do parto, peso ao nascer do recémnascido, aspectos do pré-natal e indicadores sócio-econômicos, apresentam concentrações de sódio e potássio dentro ou fora da faixa de normalidade. Considerou-se dentro da faixa de

Associação entre as concentrações de sódio e potássio no colostro das nutrizes e as variáveis maternas peso ao nascer e situação sócio-econômica.

\begin{tabular}{|c|c|c|c|c|c|c|c|c|}
\hline \multirow[t]{2}{*}{ Variáveis } & \multicolumn{3}{|c|}{ Sódio } & \multicolumn{5}{|c|}{ Potássio } \\
\hline & $\begin{array}{c}5 \mathrm{a} \\
85 \mathrm{mmol} / \mathrm{L}\end{array}$ & $\begin{array}{c}<5 \mathrm{e}> \\
85 \mathrm{mmol} / \mathrm{L}\end{array}$ & $\chi^{2}$ & $\mathrm{p}$ & $\begin{array}{c}12- \\
25 \mathrm{mmol} / \mathrm{L}\end{array}$ & $\begin{array}{l}<12 \mathrm{e}> \\
25 \mathrm{mmol} / \mathrm{L}\end{array}$ & $\chi^{2}$ & $\mathbf{P}$ \\
\hline \multicolumn{9}{|l|}{ Hipertensa } \\
\hline Sim & 17 & 16 & $1, .27$ & 0,260 & 24 & 9 & 0,01 & 0,911 \\
\hline Não & 47 & 25 & & & 50 & 22 & & \\
\hline \multicolumn{9}{|l|}{ Adulta } \\
\hline Sim & 43 & 35 & 3,42 & 0,064 & 60 & 18 & 4,91 & 0,027 * \\
\hline Não & 21 & 6 & & & 14 & 13 & & \\
\hline \multicolumn{9}{|l|}{ Primípara } \\
\hline Sim & 32 & 13 & 2,71 & 0,099 & 29 & 16 & 0,92 & 0,339 \\
\hline Não & 32 & 28 & & & 45 & 15 & & \\
\hline \multicolumn{9}{|c|}{ Mãe hipertensa ** } \\
\hline $\operatorname{Sim}$ & 33 & 20 & 0 & 0,989 & 37 & 16 & 0,01 & 0,916 \\
\hline Não & 29 & 19 & & & 34 & 14 & & \\
\hline \multicolumn{9}{|l|}{ Pai hipertenso } \\
\hline Sim & 25 & 7 & 4,55 & $0,033 * \star \star$ & 23 & 9 & 0 & 0,998 \\
\hline Não & 37 & 32 & & & 48 & 21 & & \\
\hline \multicolumn{9}{|c|}{ Peso do recém-nascido } \\
\hline Normal & 50 & 31 & 0 & 0,951 & 60 & 21 & 1,51 & 0,219 \\
\hline Baixo & 14 & 10 & & & 14 & 10 & & \\
\hline \multicolumn{9}{|c|}{ Idade gestacional } \\
\hline Pré-termo & 10 & 8 & 0,06 & 0,802 & 12 & 6 & 0,01 & 0,916 \\
\hline A termo & 54 & 33 & & & 62 & 25 & & \\
\hline \multicolumn{9}{|l|}{ Pré-natal } \\
\hline Sim & 61 & 40 & 0 & 0,948 & 71 & 30 & 0,13 & 0,661 \\
\hline Não & 3 & 1 & & & 3 & 1 & & \\
\hline \multicolumn{9}{|c|}{ Orientações sobre aleitamento materno } \\
\hline Sim & 60 & 39 & 0,02 & 0,892 & 70 & 29 & 0,06 & 0,575 \\
\hline Não & 4 & 2 & & & 4 & 22 & & \\
\hline \multicolumn{9}{|c|}{ Renda (salário mínimo) ** } \\
\hline$<1$ & 25 & 12 & 5,33 & 0,069 & 28 & 9 & 2,18 & 0,336 \\
\hline $1-2$ & 22 & 9 & & & 19 & 12 & & \\
\hline$>2$ & 15 & 18 & & & 25 & 8 & & \\
\hline \multicolumn{9}{|l|}{ Procedência } \\
\hline Urbana & 44 & 28 & 0,03 & 0,868 & 48 & 24 & 1,7 & 0,301 \\
\hline Rural & 20 & 13 & & & 26 & 7 & & \\
\hline
\end{tabular}

p: nível de significância estatística do teste do $\chi^{2}$, sendo $\mathrm{p}<0,05$ indicativo de significância estatística; OR: odds ratio; IC: intervalo de confiança é estatisticamente significativo no nível de $p<0,05$ quando 1 não estiver dentro do intervalo.

* $\mathrm{OR}=3,10(1,11-8,69)$.

** 3,8\% (4) das nutrizes não souberam informar acerca da história familiar de hipertensão arterial sistêmica e renda ou relataram renda inexistente.

$\star \star \star$ OR $=3,09(1,07-9,18)$. 
normalidade valores de sódio igual ou superior a $5 \mathrm{mmol} / \mathrm{L}$ e igual ou inferior a $85 \mathrm{mmol} / \mathrm{L}$; para o potássio, considerou-se como normalidade valores iguais ou superiores a $12 \mathrm{mmol} / \mathrm{L}$ e iguais ou inferiores a $25 \mathrm{mmol} / \mathrm{L}$. Observou-se que a história familiar paterna de hipertensão se associava significativamente às concentrações de sódio no colostro, sendo que as nutrizes filhas de pai hipertenso foram as mais prováveis de possuir concentrações de sódio no colostro de 48 horas fora da faixa de normalidade. Observouse também que a idade das nutrizes associou-se significativamente às concentrações de potássio, sendo que as nutrizes adolescentes foram as mais prováveis de apresentarem concentrações de potássio no colostro de 48 horas fora da faixa de normalidade.

As médias de pressão arterial sistólica (PAS) e pressão arterial diastólica (PAD) à internação, no grupo das nutrizes normotensas e hipertensas, são mostradas na Tabela 4 e foram significativamente diferentes entre os dois grupos, sendo que as médias das pressões arteriais das nutrizes hipertensas foram superiores a 140/90mmHg. As médias de PAS e PAD de coleta no grupo das nutrizes hipertensas foram significativamente diferentes daquelas de internação no mesmo grupo, apontando para uma redução significativa dos valores obtidos à internação, como pode ser visto na Tabela 4

As médias das concentrações de sódio e potássio do colostro de nutrizes normotensas e hipertensas não apresentaram diferenças significativas. O mesmo foi observado quando se compararam as médias da razão sódio/potássio no colostro de 48 horas das nutrizes dos dois grupos (Tabela 5). Também não houve diferença estatística das concentrações de sódio e potássio no colostro em função das diferentes variáveis clínicas e epidemiológicas das nutrizes.

\section{Discussão}

As nutrizes envolvidas no estudo foram provenientes da clínica obstétrica da Sociedade Beneficente São Camilo, Hospital Monsenhor Horta, em Mariana, Minas Gerais, Brasil, no período de

Pressão arterial sistólica (PAS; média \pm DP) e pressão arterial diastólica (PAD; média \pm DP) das nutrizes normotensas e hipertensas, aferidas nos dias de internação e coleta de colostro.

\begin{tabular}{|c|c|c|c|c|}
\hline & \multicolumn{2}{|c|}{ Internação } & \multicolumn{2}{|c|}{ Coleta } \\
\hline & PAS & PAD & PAS & PAD \\
\hline & Média \pm DP & Média \pm DP & Média \pm DP & Média \pm DP \\
\hline Normotensas & $111,39 \pm 9,54(110,0)$ ax & $70,07 \pm 9,66(70)$ a1 & $109,31 \pm 10,12(110) a x$ & $69,31 \pm 9,39(70) \mathrm{a} 1$ \\
\hline Hipertensas & $159,39 \pm 26,21(160) b x$ & $103,03 \pm 19,76(100) b 1$ & $137,58 \pm 21,36(130)$ by & $89,70 \pm 14,25(90) b 2$ \\
\hline
\end{tabular}

Legenda: x e y: comparação das médias de PAD de internação e coleta, nas linhas; 1 e 2: comparação das médias de PAS e PAD de internação e coleta, nas linhas; a e b: comparação das médias de PAS e PAD de internação e coleta, nas colunas .

Nota: valores entre parêntese representam as respectivas medianas de pressão arterial; letras ou números diferentes indicam valores estatisticamente diferentes, sendo $p \leq 0,05$.

Concentrações ( $\mathrm{mmol} / \mathrm{L} ;$ média \pm DP) de sódio e potássio e a razão sódio/potássio no colostro das nutrizes normotensas e hipertensas.

\begin{tabular}{lccc}
\hline & $\begin{array}{c}\text { Sódio } \\
\text { Média } \pm \text { DP }\end{array}$ & $\begin{array}{c}\text { Potássio } \\
\text { Média } \pm \text { DP }\end{array}$ & $\begin{array}{c}\text { Sódio/Potássio } \\
\text { Média } \pm \text { DP }\end{array}$ \\
\hline Normotensas & $87,0 \pm 14,3(85,0) a$ & $14,5 \pm 4,6(13,8) b$ & $6,8 \pm 3,0(6,5) c$ \\
Hipertensas & $87,8 \pm 11,8(85,0) a$ & $14,4 \pm 4,2(14,2) b$ & $6,8 \pm 2,7(6,0) c$ \\
\hline
\end{tabular}

Notas: valores entre parênteses representam as medianas das respectivas concentrações de eletrólitos no colostro; letras diferentes em uma mesma coluna indicam médias estatisticamente diferentes (teste $t ; p \leq 0,05$ ). 
junho a novembro de 2003. O colostro foi coletado por expressão manual segundo técnica relatada por Vinha 7 . As amostras de colostro foram preparadas e analisadas segundo recomendações de Keenan et al. ${ }^{8}$, que afirmaram não existir diferença significativa entre os valores encontrados para o sódio e o potássio no leite humano, quando obtidos por fotometria de chama ou utilizando-se a digestão ácida seguida de espectrofotometria de absorção atômica.

Dados do presente estudo mostraram associação significativa das variáveis maternas idade e paridade, à condição de hipertensão arterial na gestação, ocorrendo uma maior prevalência de hipertensão em gestantes adultas e multíparas. Segundo Saunders 9 e Cabral 10, a ocorrência de DHEG é preferencialmente em adolescentes e primíparas. Outrossim, os achados do presente estudo estão em concordância com aqueles de Sibai et al. 11, que encontraram um grande porcentual de multíparas $(75,3 \%)$ dentre as 761 gestantes hipertensas.

Considerando-se a variável história familiar de hipertensão, esta não apresentou associação positiva com a hipertensão na gestação, entretanto pode-se observar que a prevalência de hipertensão na gestação foi duas vezes maior em nutrizes filhas de mães hipertensas que naquelas filhas de mães normotensas e filhas de pais hipertensos. Prata-Martins 12 relatou que há uma tendência familiar para DHEG e Qiu et al. ${ }^{13}$ concluíram que mulheres com parentes maternos e paternos com história de hipertensão tinham um risco aumentado para DHEG. Nossos achados confirmam a influência genética na gênese da HAS.

Quando foram testadas as associações entre o peso ao nascer do recém-nascido e a idade gestacional na ocasião do parto com a hipertensão na gestação, não encontraram-se resultados significativos. Além disso, não se encontrou diferença significativa entre as prevalências de baixo peso e prematuridade nos grupos de nutrizes hipertensas e normotensas. Kalhale et al. 14 relatam que a hipertensão materna pode ser uma condição para baixo peso ao nascer e prematuridade, e Nascimento et al. 15 relataram que outros fatores além da hipertensão arterial na gestação (outros filhos com baixo peso ao nascer, grande multiparidade, desnutrição materna, que não foram investigados no presente estudo) podem contribuir para o baixo peso ao nascer e/ou prematuridade.

Analisando-se as variáveis sócio-econômicas, estas não associaram-se significativamente à história de hipertensão das nutrizes, ao contrário dos relatos de Saunders 9 e Dórea \& Lotufo 16 que afirmaram estar as diferenças sócio-econômicas (renda familiar, escolaridade, estado marital e outros) implicadas na alta prevalência da HAS. Acredita-se que a participação da maioria das gestantes $(96,2 \%)$ no pré-natal tenha minimizado o efeito negativo das condições sócio-econômicas desfavoráveis na gênese da hipertensão arterial na gestação, ao ponto de não mostrar uma associação significativa.

Quando foram testadas as associações das variáveis maternas, incluindo a condição de ter apresentado ou não a hipertensão na gestação, com as concentrações de sódio e potássio no colostro de 48 horas, observou-se que a variável idade influenciou as concentrações de potássio no colostro de 48 horas ( $\mathrm{p} \leq 0,05)$, sendo as nutrizes adolescentes três vezes mais prováveis de apresentarem concentrações de potássio no colostro fora da faixa de normalidade $(\mathrm{OR}=3,88$; IC95\%: $1,07-9,18)$. Justificativas para esse achado poderiam nortear os relatos de Keenan et al. ${ }^{\text {, que }}$ concluíram que a dieta materna pode influenciar os níveis pós-prandiais de potássio no leite materno. Assim, considerando-se que a maioria das adolescentes possui um pobre padrão alimentar, principalmente com relação ao consumo de frutas e vegetais que, com poucas exceções, são fontes alimentares ricas em potássio, o colostro poderia ter concentrações inapropriadas deste nutriente. Para controlar esse possível viés, as dietas das nutrizes foram normais quanto aos teores de sódio e potássio no puerpério imediato. Entretanto, deve-se considerar que o colostro é produzido a partir do terceiro trimestre da gestação ${ }^{17}$, quando não se teve nenhum controle sobre a dieta da gestante adolescente, o que pode ter favorecido a presença de concentrações inapropriadas de potássio no colostro das nutrizes adolescentes.

Pode-se observar também (Tabela 3) que a paridade não associou-se significativamente às concentrações de sódio e potássio no colostro, o que corrobora, em parte, os achados de Rodriguez et al. 18 e Alaudeen et al. 19. Estes, estudando as concentrações de sódio no leite materno do $1^{\circ}$ ao $90^{\circ}$ dia pós-parto, afirmaram que a paridade não afetou significativamente o nível de sódio no leite humano.

Quanto á história familiar de hipertensão, observou-se que a variável história paterna de hipertensão associou-se às concentrações de sódio no colostro de 48 horas ( $p<0,05$ ), sendo que as nutrizes filhas de pais hipertensos foram as mais prováveis de apresentarem concentrações de sódio fora da faixa de normalidade $(\mathrm{OR}=3,09$; IC95\%: 1,07-9,18). Esperava-se uma possível relação das concentrações de sódio fora da faixa de normalidade para filhas de mães hipertensas, ou de ambos os pais hipertensos, corroborando 
a influência genética da hipertensão materna ou de ambos os pais hipertensos sobre a nutriz. Para testar a possível influência da variável "mãe hipertensa" no resultado encontrado, realizou-se uma análise estatística multivariada, não encontrando associação nem mesmo com a variável "pai hipertenso". Possivelmente esse achado se confirmaria se a amostra de nutrizes com história familiar de hipertensão fosse maior. Também não se observaram associações entre a história familiar materna de hipertensão com as concentrações de sódio no colostro de 48 horas das nutrizes do estudo.

A idade gestacional e peso do recém-nascido não associaram-se significativamente às concentrações de sódio e potássio no colostro. Gross et al. ${ }^{5}$ relataram que as concentrações de sódio foram significativamente mais altas no leite de nutrizes que tiveram bebês pré-termo, que no leite daquelas que tiveram bebês a termo. Porém, Weffort 20 também não encontrou diferenças nas concentrações de sódio nos leites de nutrizes que tiveram bebês a termo ou pré-termo, estando os dados do presente estudo em concordância com aqueles de Weffort 20 .

Quando foram comparadas as médias das concentrações de sódio e potássio no colostro das nutrizes normotensas e hipertensas, estas não mostraram diferenças significativas (Tabela 5). Neville et al. 21 encontraram valores para o sódio nas primeiras 60 horas pós-parto, que variaram de 15 a $80 \mathrm{mmol} / \mathrm{L}$, sendo o maior valor encontrado nas primeiras horas pós-parto, devido muito provavelmente, segundo os autores, à permanência da via paracelular no início do processo de lactação. Grumash et al. ${ }^{4}$ estudando os fatores nutricionais em colostro de mães brasileiras encontraram valores de sódio que variaram de 5 a $85 \mathrm{mmol} / \mathrm{L}$ nas primeiras 48 horas pósparto. No presente estudo, as médias de sódio no colostro de 48 horas das nutrizes normotensas e hipertensas foram superiores aos valores relatados por Neville et al. 21 e Grumash et al. 4, não havendo distinção entre aquelas de nutrizes normotensas e hipertensas. Ereman et al. 22 afirmaram que, dos minerais no leite humano, a concentração de sódio é a mais variável durante toda a lactação, e Wack et al. 23 relataram existir variações intra e inter-indivíduos quanto à secreção deste eletrólito no leite humano. A existência dessas variações inter e intra-individuais na composição do leite humano poderiam justificar, em parte, as diferenças encontradas entre as concentrações de sódio do presente estudo e os dados da literatura. Além disso, observaramse diferentes delineamentos metodológicos na literatura, o que prejudica a análise comparativa entre os dados.
Quanto ao potássio, Woisky 24 encontrou uma média no colostro igual a $13 \mathrm{mmol} / \mathrm{L}$. Wack et al. 23 encontraram uma média de 585,0 $\pm 124,0 \mathrm{mg} / \mathrm{L}(14,96 \mathrm{mmol} / \mathrm{L})$ de potássio em 140 amostras de leite de 30 nutrizes nos primeiros 60 dias pós-parto, e afirmaram existir maior variabilidade inter-individual com relação aos níveis de potássio no leite humano, em comparação aos níveis de sódio. As médias de potássio no colostro das nutrizes normotensas e hipertensas deste estudo não diferiram estatisticamente, e foram ligeiramente superiores à média encontrada por Woisky 24 e bastante próximas àquela de Wack et al. 23. Gross et al. 5 investigando a composição do leite de mulheres que tiveram seus bebês a termo e pré-termo encontraram valores de potássio variando 12 a $25 \mathrm{mmol} / \mathrm{L}$, e afirmaram que as concentrações de potássio no leite dos diferentes grupos de nutrizes foram similares. Neville et al. 21 encontraram uma redução nos níveis de potássio nos primeiros dias pós-parto, sendo as médias de potássio no dia do nascimento igual a $18 \mathrm{mmol} / \mathrm{L}$ e no 20 o dia pós-parto igual a $14 \mathrm{mmol} / \mathrm{L}$. As médias de potássio no colostro das nutrizes normotensas e hipertensas do presente estudo estão próximas à média relatada por Wack et al. 23 e se encontram dentro da faixa de variação encontrada por Gross et al. 5. As aparentes diferenças entre as médias de potássio do presente estudo e as encontradas nos outros estudos citados vêm confirmar as variações inter e intra-individuais das concentrações de potássio no leite humano, relatadas por Wack et al. 23 .

Ressalta-se que houve uma significativa redução das médias das pressões arteriais sistólica e diastólica das nutrizes hipertensas no dia da coleta do colostro, atingindo a faixa de normalidade (Tabela 4), conforme observações de Kalhale et al. 14, o que pode justificar, em parte, a ausência de significância estatística entre as concentrações de sódio e potássio e da razão $\mathrm{Na} / \mathrm{K}$ no colostro das nutrizes normotensas e hipertensas (Tabela 5). Fraxino et al. 25 descrevem os diferentes mecanismos para a regulação da pressão arterial (cardíacos, renais, neurais, hormonais vasoconstritores e hormonais vasodilatadores). Assim, pequenas diferenças nas concentrações de sódio e potássio no colostro de nutrizes normotensas e hipertensas seriam esperadas caso houvesse alguma falha nos mecanismos que regulam a pressão arterial, resultando na elevação das concentrações destes eletrólitos no plasma. Assim, através da via paracelular, uma das vias de secreção de componentes do colostro no período gestacional que permite livre fluxo de sódio e potássio da corrente sangüínea para o lúmen das células alveolares da glândula mamária 17, o sódio e o potássio passariam livremente para o 
colostro das nutrizes hipertensas em concentrações diferentes daquelas encontradas no colostro de nutrizes normotensas. Contudo, Guyton \& Hall 26 afirmaram que o organismo dispõe de sensíveis mecanismos que impedem qualquer pequeno aumento de sódio e potássio no plasma. Possivelmente, em uma amostra mais expressiva quanto ao número de nutrizes normotensas e hipertensas, pequenas diferenças nas concentrações de sódio e potássio no colostro de 48 horas poderiam ser encontradas.

\section{Conclusões}

Os resultados do presente estudo mostraram que, estatisticamente, as concentrações de sódio e potássio no colostro de 48 horas de nutrizes normotensas e hipertensas não foram diferentes. Em contra partida, a pressão arterial das nutrizes hipertensas se normalizou nos primeiros dias do puerpério, podendo ser este o fator determinante da similaridade das concentrações dos eletrólitos no colostro das nutrizes normotensas e hipertensas.

Apenas as variáveis clínicas idade e paridade associaram-se significativamente com a condi- ção de hipertensão das nutrizes, e as adolescentes foram as mais prováveis de apresentarem valores de potássio fora da faixa de normalidade, provavelmente em função de uma dieta pobre em potássio durante o último trimestre de gestação.

As variáveis epidemiológicas das nutrizes (freqüência ao pré-natal, acesso a orientações para o sucesso do aleitamento materno, renda familiar e procedência) não se associaram com a condição da nutriz de ser ou não hipertensa, bem como não interferiram nas concentrações de sódio e potássio no colostro de 48 horas das nutrizes do estudo.

Por bem, a hipótese de que a amamentação precoce de filhos de nutrizes hipertensas poderia expor o recém-nascido a quantidades inapropriadas de sódio e potássio, não foi confirmada neste estudo.

Conclui-se que a prática de aleitamento materno por nutrizes hipertensas provavelmente não aumenta o risco de seus filhos desenvolverem a hipertensão arterial na idade adulta. Portanto, a influência da história de hipertensão na gestação no desenvolvimento da hipertensão arterial sistêmica crônica provavelmente não se dá através do aleitamento materno precoce.

\section{Resumo}

O objetivo do presente trabalho foi avaliar se a hipertensão arterial e outras variáveis clínicas e epidemiológicos influenciam as concentrações de sódio e potássio no colostro de 48 horas de nutrizes. Para tal, contou-se com a participação de 105 nutrizes, sendo 72 (68,6\%) normotensas e 33 (31,4\%) hipertensas. Coletou-se uma única amostra de colostro de 48 horas de cada nutriz, no período da manhã. As amostras foram analisadas em fotômetro de chama para determinação das concentrações de sódio e potássio. Quando se testaram as associações das concentrações de sódio e potássio com as variáveis: idade, paridade, história familiar de hipertensão, idade gestacional, peso do recém-nascido e fatores socioeconômicos, encontrou-se associação significativa da idade das nutrizes com a concentração de potássio no colostro. As médias das concentrações de sódio e potássio no colostro das nutrizes normotensas e hipertensas não apresentaram diferenças significativas. Portanto, a prática de aleitamento materno por nutrizes hipertensas provavelmente não aumenta o risco de seus filhos desenvolverem a hipertensão arterial na idade adulta.

Colostro; Hipertensão; Sódio; Potássio

\section{Colaboradores}

M. A. O. Sírio foi responsável pela autoria do projeto, coleta e análise dos dados e redação do texto. M. E. Silva foi responsável pela autoria do projeto, análise dos dados e supervisão do estudo. H. Paula orientou a montagem do banco de dados e análises posteriores. M. C. Passos analisou os dados e revisou o texto. A. O. Souza Sobrinho auxiliou na padronização do método e realização das determinações de sódio e potássio nas amostras de colostro. 


\section{Referências}

1. Hofman A, Hazebroek A, Valkenburg A. A randomized trial of sodium intake and blood pressure in newborn infants. JAMA 1987; 250:370-3.

2. Holliday M. O teor de sal nos primeiros alimentos afeta a pressão sangüínea em etapas posteriores da vida? [sumário]. In: Nestlé Nutrition Service - 36 Seminário Nestlé Nutrion. Vevey: Nestec S.A.; 1997. p. 23-6

3. Salgado CM, Carvalhaes JTA. Hipertensão arterial na infância. J Pediatr (Rio de J) 2003; 79 Suppl l: S115-24.

4. Grumash AS, Jerônimo SEI, Hage M, CarneiroSampaio MMS. Nutritional factors in milk from brazilian mothers delivering small for gestacional age neonates. Rev Saúde Pública 1993; 27:455-62.

5. Gross SJ, Geller JY, Tomarelli RM. Composition of breast milk from mothers of preterm infants. Pediatrics 1981; 68:490-3.

6. Organización Mundial de la Salud. El embarazo y aborts em la adolescencia. Geneve: Organización Mundial de la Salud; 1975. (Série de Informes Técnicos, 583).

7. Vinha VHP. Amamentação materna, incentivo e cuidados. São Paulo: Sarvier Editora; 1987.

8. Keenan BS, Buzek SW, Garza C, Potts E, Nichols BL. Diurnal and longitudinal variations in human milk sodium and potassium: implication for nutrition and physiology. Am J Clin Nutr 1982; 35:527-34.

9. Saunders C. Síndromes hipertensivas da gravidez - SHG. In: Accioly E, Saunders C, Lacerda EMA, organizadores. Nutrição em obstetrícia e pediatria. 2a Ed. Rio de Janeiro: Cultura Médica; 2002. p. 189207.

10. Cabral ACV. Manual de rotinas em obstetrícia: protocolo de conduta da maternidade do Hospital Odilon Behrens. Belo Horizonte: Prefeitura Municipal de Belo Horizonte; 1996

11. Sibai BM. Chronic hypertension in pregnancy. Obstet Gynecol 2002; 100:369-77.

12. Prata-Martins JA. Gestoses. In: Prata-Martins JA, organizador. Patologia obstétrica. São Paulo: Editora Roca; 1985 . p. 84-96.

13. Qiu C, Williams MA, Leisering WM, Sorensen TK, Frederick IO, Dempsey JC, et al. Family history of hypertension and type 2 diabetes in relation to preeclampsia risk. Hypertension 2003; 41:408-13.

14. Kalhale S, Zugaib M, Neme B. Doença hipertensiva específica da gestação - aspectos fisiopatológicos e etiopatogênicos. In: Neme B, organizador. Patologia da gestação. São Paulo: Sarvier Editora; 1988. p. 145-8.
15. Nascimento LFC. Estudo transversal sobre fatores associados ao baixo peso ao nascer a partir de informações obtidas na sala de vacinação. Rev Bras Saúde Materno Infantil, 2003; 3:37-42.

16. Dórea EL, Lotufo PA. Epidemiologia da hipertensão arterial sistêmica. Rev Brasileira de Hipertensão 2004; 7:86-93.

17. Lawrence RA, Lawrence RM. Biochemistry of human milk. In: Lawrence RA, Lawrence RM, editor. Breastfeeding: a guide for the medical profession. 5a Ed. New York: Mosby; 1999. p. 95-158.

18. Rodriguez REM, Alaejos MS, Romero CD. Concentraciones de calcio, magnesio y potasio en leche materna y fórmulas de inicio. Arq Latinoameric Nutr 2002; 52:406-12.

19. Alaudeen S, Muslim N, Faridah K, Azman A, Arshat H. Eletrolyte profile of Malaysian mother's milk. Malays J Reprod Health 1988; 6:102-7.

20. Weffort VRS. Concentração de nitrogênio total, sódio, cloro, cálcio, fósforo, magnésio, potássio, cobre e zinco no leite de mães de recém-nascidos a termo e pré-termo [Dissertação de Mestrado]. São Paulo: Faculdade de Medicina de Ribeirão Preto, Universidade de São Paulo; 1998.

21. Neville MC, Allen JC, Archer PC, Casey CE, Seacot J, Keller RP, et al. Studies in human lactation: milk volume and nutrient composition during weaning and lactogenesis. Am J Clin Nutr 1991; 54:81-92.

22. Ereman, RR, Lonnerdal BO, Dewey KG. Maternal sodium intake does not affect postprandial sodium concentration in human milk. J Nutrition 1987; 117:1154-7.

23. Wack RP, Lien EC, Taft D, Roscelli JD. Eletrolyte composition of human breast milk beyond the early postpartum period. Nutrition 1997; 13:774-7.

24. Woisk JR. Nutrição e dietética em pediatria. 4a Ed. São Paulo: Editora Atheneu; 1995.

25. Fraxino PH, Martins C, Riella MC. Nutrição na hipertensão arterial. In: Riella, MC, Martins C, organizadores. Nutrição e o rim. Rio de Janeiro: Editora Guanabara Koogan; 2001. p. 953-7.

26. Guyton AC, Hall JE. Regulação neural da circulação e o controle rápido da pressão arterial. In: Gutton AC, Hall JE, organizadores. Tratado de fisiologia médica. 9a Ed. Rio de Janeiro: Editora Guanabara Koogan; 1997. p. 191-211.

Recebido em 24/Ago/2005

Versão final reapresentada em 13/Set/2006 Aprovado em 13/Mar/2007 